\title{
Extensions of Guiding Center Motion to Higher Order
}

\author{
Theodore G. Northrop \\ James A. Rome
}

OAK RIDGE NATIONAL LABORATORY 


\section{DISCLAIMER}

This report was prepared as an account of work sponsored by an agency of the United States Government. Neither the United States Government nor any agency Thereof, nor any of their employees, makes any warranty, express or implied, or assumes any legal liability or responsibility for the accuracy, completeness, or usefulness of any information, apparatus, product, or process disclosed, or represents that its use would not infringe privately owned rights. Reference herein to any specific commercial product, process, or service by trade name, trademark, manufacturer, or otherwise does not necessarily constitute or imply its endorsement, recommendation, or favoring by the United States Government or any agency thereof. The views and opinions of authors expressed herein do not necessarily state or reflect those of the United States Government or any agency thereof. 


\section{DISCLAIMER}

Portions of this document may be illegible in electronic image products. Images are produced from the best available original document. 


\title{
Printed in the United States of America. Available from National Technical Information Service U.S. Department of Commerce 5285 Port Royal Road, Springfield, Virginia 22161 \\ Price: Printed Copy $\$ 4.00$; Microfiche $\$ 3.00$
}

\begin{abstract}
This report was prepared as an account of work sponsored by the United States Government. Neither the United States nor the Energy Research and Development Administration/United States Nuclear Regulatory Commission, nor any of their employees, nor any of their contractors, subcontractors, or their employees, makes any warranty, express or implied, or assumes any legal liability or responsibility for the accuracy, completeness or usefulness of any information, apparatus, product or process disclosed, or represents that its use would not infringe privately owned rights.
\end{abstract}


ORNL/TM-5952

Contract No. W-7504-eng-26

Fusion Energy Division

EXTENSIONS OF GUIDING CENTER MOTION TO HIGHER ORDER

Theodore G. Northrop

NASA/Goddard Space Flight Center, Greenbelt, Maryland 20771

\author{
James A. Rome
}

Oak Ridge National Laboratory, Oak Ridge, Tennessee 37830

Date Published: July 1977

Prepared by the OAK RIDGE NATIONAL LABORATORY

Oak Ridge, Tennessee 37830

operated by

UNION CARBIDE CORPORATION for the

ENERGY RESEARCH AND DEVELOPMENT ADMINISTRATION

NOTICE This document contains information of a preliminary nature. It is subject to revision or correction and therefore does not represent a final report.
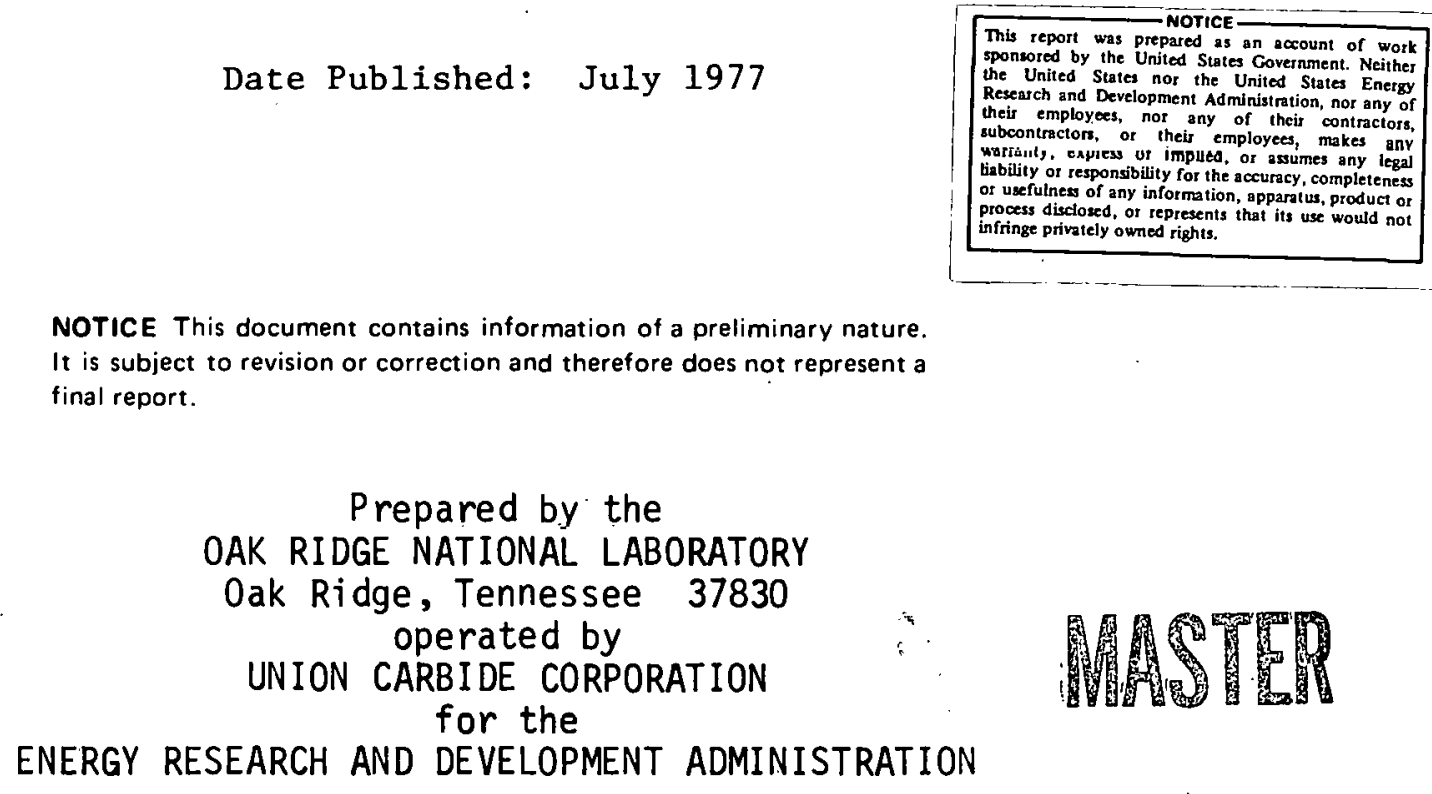
EXTENSIONS OF GUIDING CENTER MOTION TO HIGHER ORDER

Theodore G. Northrop

NASA/Goddard Space Flight Center, Greenbelt, Maryland 20771

James A. Rome

Oak Ridge National. Laboratory, Oak Ridge, Tennessee 37830

\section{ABSTRACT}

In a static magnetic field, some well-known guiding center equations maintain their form when extended to next order in gyroradius. In these cases, it is only necessary to include the next order term in the magnetic moment series. The differential equation for guiding center motion which describes both the parallel and perpendicular velocities correctly through first order in gyroradius is given. The question of how to define the guiding center position through second order arises and is discussed, and second order drifts are derived for one usual definition.

The toroidal canonical angular momentum, $P_{\phi}$, of the guiding center in an axisymmetric field is shown to be conserved using the guiding center velocity correct through first order. When second order motion is included, $P_{\phi}$ is no longer a constant.

The above extensions of guiding center theory help to resolve the different tokamak orbits obtained either by using the guiding center equations of motion or by using conservation of $P_{\phi}$. 


\section{INTRODUCTION}

Nowadays, guiding center theory is routinely used in various plasma calculations. This can usually be done without being too careful about whether quantities are being evaluated at the instantaneous particle position or at the guiding center or without being concerned with second order effects in gyroradius. However, a disagreement between two methods for obtaining certain tokamak orbits has led us to critically reexamine guiding center theory and to extend much of it through second order in gyroradius. While doing this, we have discovered in the literature various mistakes and unclarities, which we have tried to correct. Because the results to be presented in the main body of the paper are general and not limited to tokamaks, we describe the tokamak orbit problem in Appendix I.

\section{HIGIIER ORDCR LFFECTS}

It is well known' that in a static magnetic field (no electric field) the component $v_{\|}$of guiding center velocity parallel to the magnetic field $\vec{B}(\vec{R})$ at the guiding center $\vec{R}$ obeys $\frac{d}{d t}\left[v_{\|}^{2} / 2+\left(M_{0} / m\right) B(\vec{R})\right]$ $=0+O(\varepsilon)$, where $\varepsilon$ is the adiabatic parameter $\mathrm{m} / \mathrm{e}$ and is proportional to the gyroradius. $M_{0}$ is the lowest order (in gryoradius) magnetic moment $\mathrm{mv}_{1}{ }^{2} / 2 \mathrm{~B}$. It is perhaps less well known that this energy integral holds to one higher order, provided $M_{0}$ is replaced by the sum of the first two terms $\left(M_{0}+\varepsilon M_{1}\right)$ of the magnetic moment series. 


$$
\begin{aligned}
& \frac{d}{d t}\left[\frac{v_{\|}^{2}}{2}+\frac{\left(M_{0}+\varepsilon M_{1}\right)}{m} B(\vec{R})\right]=0+0\left(\varepsilon^{2}\right) \\
& \frac{v_{\|}^{2}}{2}+\frac{\left(M_{0}+\varepsilon M_{1}\right)}{m} B(\vec{R})=\frac{K}{m}+0\left(\varepsilon^{2}\right) .
\end{aligned}
$$

$K$ is the energy to this order. $M_{1}$ was derived by Kruskal ${ }^{2}$ and is given in several forms in Ref. 1. In a static magnetic field

$$
\begin{aligned}
& M_{0}+\varepsilon M_{1}= \\
& \frac{m v_{1}^{2}}{2 B(\vec{r})}-\frac{m^{2}}{2 e B^{3}}\left\{\left(v^{2} \hat{L}+\overrightarrow{v v} \cdot \vec{L}\right) \cdot[(\vec{v} \times \hat{L}) \cdot \nabla \vec{B}]+\vec{v} \cdot \hat{L}(\nabla \times \vec{B}) \cdot\left(\frac{v_{1}^{2}}{2} \hat{L}+2 \vec{v} \vec{v} \cdot \hat{L}\right)\right\},
\end{aligned}
$$

where $\hat{L}=\vec{B} / B$ and $\vec{V}_{\perp}$ is the component of particle velocity perpendicular to the field $\vec{B}(\vec{r})$ at the particle position $\vec{r}$. Equation (1) was derived (incorrectly) by Northrop, Liu, and Kruskal. ${ }^{3}$ Wilson ${ }^{4}$ pointed out the algebraic error, ${ }^{*}$ and Eq. (21) of Ref. 3, when corrected, becomes precisely Eq. (1).

The three coupled differential equations in Ref. 3 governing guiding center motion then can be shown to give

$$
\begin{aligned}
\frac{d \vec{R}}{d t}= \pm\left(\frac{2}{m}\right)^{1 / 2}\left[K-\left(M_{U}+\varepsilon M_{1}\right) B\right]^{1 / 2} \hat{L}(\vec{R}) & +\frac{m}{e B} \frac{2}{m}(K-M B) \hat{L} x \hat{L} \cdot \nabla \hat{L} \\
& +\frac{M}{e B} \hat{L} x \nabla B,
\end{aligned}
$$

where all field quantities are at $\vec{R}$, and $M=M_{0}+\varepsilon M_{1}$. Equations (1)-(3) replace the three quite complicated coupled differential equations.

\footnotetext{
* The error produced minor changes in some of the expressions of Ref. 3 , although it did not affect the first correction to the longitudinal invariant. Expressions in that reference should not be used wi thout checking with an author (T.G.N.).
} 
Morozov and Solov'ev 5 have given similar coupled differential equations, but did not take advantage of the constants of the motion (1) and (2) to put the equation of motion in the form of (3) in a static field. It is remarkable that the use of $\varepsilon M_{1}$ as in (3) is all that is necessary to treat the parallel guiding center velocity correctly through first order in gyroradius (i.e., $\varepsilon$ ). The order $\varepsilon$ term of the parallel velocity is sometimes called "parallel drift." The expression for it depends on what one has chosen for the lowest order parallel velocity, and caution is called for in using the parallel velocities seen in the literature. There is no analogous ambiguity for the perpendicular drifts because their lowest order is al ready first order in $\varepsilon$.

In practice when one integrates (3) numerically, he chooses a number for the magnetic moment and holds it constant during the integration. And this is exactly what one would do in one lower order, solving $v_{\|}^{2} / 2$ $+M_{0} B=$ constant for the parallel velocity. The only difference is that in order to know what number to assign as $M$ for a particle whose initial position and velocity are given, the expressions for $M_{0}$ and $M_{1}$ must be used.

Baños $^{6}$ has a different expression for the parallel guiding center velocity from that in (3). The difference occurs because his lowest order parallel velocity is the time average of the component of instantaneous particle velocity parallel to the field $\vec{B}(\vec{r})$ at the particle position. As shown in Appendix II, this "average instantaneous parallel velocity" differs from the parallel guiding center velocity, $v_{\|} \equiv \frac{d \vec{R}}{d t} \cdot \hat{L}(\vec{R})$, if the magnetic field has curl along $\vec{B}$ (i.e., $J_{\|} \neq 0$ ). The 
paralle.l guiding center velocity may even vanish and the "average instantaneous parallel velocity" not vanish. This does not mean that the guiding center takes leave of $i$ ts particle. It does not. This matter is, however, an aside to the present work, so it is discussed more fully in Appendix II.

In another situation, a higher order result follows from the lower order by the substitution of $M_{0}+\varepsilon M_{1}$ for $M_{0}$. The rate of change of the parallel guiding center velocity is well known to lowest order to be

$$
\frac{d v_{\|}}{d t}=-\frac{M_{0}}{m} \hat{L} \cdot \nabla B(\vec{R})+O(\varepsilon) .
$$

It is easy to obtain this "mirror effect" to the next order. From (3),

$$
\frac{d v_{\|}}{d t}= \pm\left(\frac{2}{m}\right)^{1 / 2} \frac{d}{d t}[K-M B(\vec{R})]^{1 / 2}=\frac{\mp M(d B / d t)}{\left(\frac{2}{m}\right)^{1 / 2}(K-M B)^{1 / 2}}+0\left(\varepsilon^{2}\right),
$$

where $d B / d t=(d \vec{R} / d t) \cdot \nabla B$. One obtains

$$
\begin{aligned}
\frac{d v_{\|}}{d t} & =-\frac{\left(M_{0}+\varepsilon M_{1}\right)}{m} \hat{L} \cdot \nabla B \pm \frac{\varepsilon M}{m B} \frac{2}{m}(K-M B)^{1 / 2}(\hat{L} \times \nabla B) \cdot(\hat{L} \cdot \nabla \hat{L})+O\left(\varepsilon^{2}\right) \\
& =\frac{-\left(M_{0}+\varepsilon M_{1}\right)}{m} \hat{L} \cdot\left[\nabla B+\frac{\varepsilon v_{\|}}{B}(\hat{L} \times \hat{L} \cdot V \hat{L}) \times(\hat{L} \times \vee B)\right]+O\left(\varepsilon^{2}\right) .
\end{aligned}
$$

The second term of (5) (containing $v_{\|}$) can easily be shown to vanish if the current density $\vec{J}$ is parallel to $\vec{B}$, so that $(\nabla \times \vec{B})_{1}$ vanishes. The component $\hat{L} \cdot d^{2} \vec{R} / d t^{2}$ of acceleration parallel to $\vec{B}$ can now be obtained to the same order as (5). The difference,

$$
\frac{d v_{\|}}{d t}-\hat{L} \cdot \frac{d^{2} \vec{R}}{d t^{2}}=\frac{d}{d t}\left(\hat{L} \cdot \frac{d \vec{R}}{d t}\right)-\hat{L} \cdot \frac{d^{2} \vec{R}}{d t^{2}}=\frac{d \hat{L}}{d t} \cdot \frac{d \vec{R}}{d t},
$$


can be obtained directly from $(3)$ because $d \hat{L} / d t=(d \hat{R} / d t) \cdot \nabla \hat{L}$. The difference just cancels the last term of (5).

$$
\hat{L} \cdot \frac{d^{2} \vec{R}}{d t^{2}}=-\frac{M_{0}+\varepsilon M_{1}}{m} \hat{L} \cdot \nabla B+O\left(\varepsilon^{2}\right) .
$$

Here again the higher order result follows from the lower order by correcting the magnetic moment. The reader should not believe, however, that all higher order results follow from corresponding lower order ones by such a simple prescription. Some do and some do not. Equations (4) and (5) are a counterexample. We do not know arly yeneral prescription for when the method works, but in this paper we show two cases where it does work.

Wi 1 son $^{4}$ has given $\frac{d v_{\mathbb{1}}}{d t}$ through order $\varepsilon$, but his result disagrees with (5). We believe (5) to be correct, and our method for obtaining it is far simpler than his. The vector algebra required to obtain some of the results in the present paper and in Refs. 3 and 4 is truly extensive and presents an almost infinite opportunity for mistakes. Rather than giving the algebra in an appendix, we prefer to offer it privately to any reader who would like to check it or, hopefully, even find easier me thods.

At this point it is apparent that the guiding center obeys an equation of the form

$$
\frac{d^{2} \vec{R}}{d t^{2}}=\frac{1}{\varepsilon} \frac{d \vec{R}}{d t} \times \vec{B}(\vec{R})-\frac{M_{0}+\varepsilon M_{1}}{m} \nabla B(\vec{R})+\varepsilon \hat{L} \times \vec{W}(\vec{R})+0\left(\varepsilon^{2}\right),
$$

where $\vec{W}$ is yet to be found.

It is already known [Ref. 1, Eq. (1.12) and Ref. 2] that 


$$
\frac{d^{2} \vec{R}}{d t^{2}}=\frac{1}{\varepsilon} \frac{d \vec{R}}{d t} \times \vec{B}(\vec{R})-\frac{M_{O}}{m} \nabla B(\vec{R})+0(\varepsilon)
$$

Furthermore, the parallel (to $\vec{B}$ ) component of (7) is indeed (6). Equation (1) follows by dotting (7) with $d \vec{R} / d t$ by the usual method for obtaining an energy integral. One must use the fact that $\varepsilon \frac{d \vec{R}}{d t} \cdot \hat{L} \times \vec{W}=\varepsilon \frac{d \vec{R}}{d t} \times \hat{L} \cdot \vec{W}$, and $\varepsilon \frac{d \vec{R}}{d t} \times \hat{L}$ is of order $\varepsilon^{2}$. The second order guiding center drifts can be obtained from (7) in terms of $\vec{W}$ by an iterative solution for $\left(\frac{d \vec{R}}{d t}\right)_{1}$. The drift velocity is

$$
\left(\frac{d \vec{R}}{d t}\right)_{1}=\frac{\varepsilon\left(M_{0}+\varepsilon M_{1}\right)}{m} \frac{\hat{L} \times \nabla B}{B}+\frac{\varepsilon}{B} \hat{L} \times \frac{d^{2} \vec{R}}{d t^{2}}+\frac{\varepsilon^{2} \vec{W}_{\perp}}{B}+0\left(\varepsilon^{3}\right),
$$

where $\vec{W}_{\perp}$ is the component of $\vec{W}$ perpendicular to $\vec{B}(\vec{R}) . \quad d^{2} \vec{R} / d t^{2}$ can be found to the order needed by time-differentiating (3) via $\frac{d^{2} \vec{R}}{d t^{2}}=\frac{d \vec{R}}{d t} \cdot \nabla \frac{d \vec{R}}{d t}$, since $\frac{d \vec{R}}{d t}$ is a function of $\vec{R}$. The result can be expressed in myriad forms, perhaps the simplest of which is

$$
\frac{d^{2} \vec{R}}{d t^{2}}=-\frac{M_{0}+\varepsilon M_{1}}{m} \nabla B+\left[\frac{\vec{u} B}{\varepsilon}\left(1-\frac{\varepsilon v_{\|} \hat{L} \cdot \nabla \times \hat{L}}{B}\right)+v_{\|}(\nabla \times \vec{u})\right] \times \hat{L}+0\left(\varepsilon^{2}\right),
$$

where $\vec{u}$ is the last two terms of (3). It is imperative here that $\vec{u}$ be calculated with $M=M_{0}+\varepsilon M_{1}$ as indicated in (3). The parallel component of (10) is (6), as it should be. Substitution of (10) into (9) gives the guiding center drift through second order in gyroradius as

$$
\left(\frac{d \vec{R}}{d t}\right)_{\perp} \equiv \vec{u}+\frac{\varepsilon v_{\|}}{B}\left[(\nabla \times \vec{u})_{\perp}-(\hat{L} \cdot \nabla \times \hat{L}) \vec{u}\right]+\frac{\varepsilon^{2} \vec{W}_{\perp}}{B}+0\left(\varepsilon^{3}\right),
$$

again with the warning that al though the $\vec{u}$ on the right side is the sum of the usual two drifts in (3), these two drifts are to be calculated using $M_{0}+\varepsilon M_{1}$ for the magnetic moment and (1) for $v_{\|}^{2}$. Thus 
$\vec{u}$ contributes in order $\varepsilon^{2}$ to the perpendicular guiding center drift velocity.

III. WHAT IS Wै?

$\vec{W}$ depends on how the guiding center position is defined. There is no arbitrariness about the guiding center definition through first order in gyroradius, hut there is in second order. For exalliple, one can define $\vec{R}$ in terms of the particle position $\vec{r}$ and velocity $\vec{v}$ by

$\vec{R}=\vec{r}+\frac{\varepsilon \vec{V} \times \vec{B}(\vec{r})}{B^{2}(\vec{r})}$

or by

$\vec{R}=\vec{r}+\frac{\varepsilon \vec{v} \times \vec{B}(\vec{R})}{B^{2}(\vec{R})}$

These two differ in $O\left(\varepsilon^{2}\right)$ and will lead to different expressions for $\vec{W}$. Both give (8). As an aside, there is in fact a corresponding arbitrariness in first order if one introduces the ronrept of a guiding point; of which the guiding center is a (very natural) special case. A guiding poinl would be defined as some function of $\vec{r}$ and $\vec{v}$ which moves smoothly, without wiggling at the gyrofrequency, and whose distance from the actual particle does not exceed a known amount. For example, the particle is always a gyroradius from its guiding center. One might choose the guiding point to be on the gyrocircle at the point where it intersects the princtpal radius of curvature of the field line. Then the particle is always within a gyrodiameter of the guiding point. This is admittedly less natural than using the guiding center, but is entirely possible. 
However, when we come to second order effects, what is natural is much less obvious. One might like to select a definition of $\vec{R}$ which leads to a $\vec{W}$ having some desired property. For example, one might like the last term of (11) to cancel the next-to-last term. Then the drifts through second order would in fact follow from the lower order drifts merely by replacing $M_{0}$ with $M_{0}+\varepsilon M_{1}$. Or one might like $\vec{W}$ to be parallel to $\vec{B}$, so that (7) has the same form as (8), but again with $M$ replacing $M_{0}$. Unfortunately, we do not know how to work the systematic adiabatic theories ${ }^{2}, 7$ backwards $-i . e .$, how to define the guiding center (or point) so as to get a desired equation of motion for it.

We have found one expression for $\vec{W}$ by defining the guiding center as Kruskal does in Ref. 2. He has solved the equation of motion of a charged particle via an asymptotic series of the form

$$
\vec{r}=\sum_{n=-\infty}^{\infty} \varepsilon^{|n| \vec{R}_{n}(t) e^{\frac{i n}{\varepsilon} \int B\left(\vec{R}_{0}\right) d t}}
$$

by equating coefficients of equal powers of $\exp \left(\frac{i}{\varepsilon} B \int d t\right)$ after substituting the series into the particle equation of motion. The resulting equations for the $\vec{R}_{n}$ 's are solvable in power series in $\varepsilon . \vec{R}_{0}$ can be taken as the guiding center position and is given in terms of $\vec{r}$ and $\vec{v}$ by

$$
\begin{aligned}
\vec{R}_{0}=\vec{r} & +\varepsilon \frac{\vec{v} \times \vec{B}(\vec{r})}{B^{2}(\vec{r})}+\varepsilon^{2}\left[\frac{-5}{4 B^{3}} v_{\perp}^{2} \nabla_{\perp} B-\frac{v_{\|}^{2}}{B^{2}} \frac{\partial \hat{L}}{\partial S}-\frac{v_{\|}}{B^{3}}(\hat{L} \cdot \nabla \times \vec{B})\left(\vec{v}_{\perp} \times \hat{L}\right)\right. \\
& \left.+2 \frac{v_{\|}}{B^{2}} \hat{L}\left(\vec{v}_{1} \cdot \frac{\partial \hat{L}}{\partial S}\right)-\frac{v_{\perp}^{2}}{8 B^{3}} \hat{L} \frac{\partial B}{\partial S}+\frac{\vec{v}_{\perp}}{2 B^{3}}\left(\vec{v}_{\perp} \cdot \nabla B\right)+\frac{3 \hat{L}}{4 B^{2}} \vec{v}_{\perp} \cdot\left(\vec{v}_{\perp} \cdot \nabla \hat{L}\right)\right]+0\left(\varepsilon^{3}\right),
\end{aligned}
$$

where $\partial / \partial s=[\cdot \nabla$. 
$\vec{R}_{0}$ is not the same in $O\left(\varepsilon^{2}\right)$ as the guiding center $\vec{p}$ of Ref. 3 . We have carried out the series to sufficient order to obtain $\vec{W}$ explicitly. This $\vec{W}$ we will denote by $\vec{W}_{1}$ to emphasize that $\vec{W}$ is not unique, even though the form of (7) is, and that it depends on how the guiding center is defined in second order:

$$
\vec{W}_{1}=\frac{v_{\|} M}{2 m B}\left[\frac{\nabla B \times(\nabla \times \vec{B})}{B}+5(\hat{L} \cdot \nabla B)(\hat{L} \cdot \nabla \hat{L})+2 B(\hat{L} \cdot \nabla \hat{L}) \cdot(\nabla \hat{C})+\nabla^{2} \vec{B}-\hat{L} \hat{L}: \nabla \nabla \vec{B}\right] \text {. }
$$

Substitution of this into (11) gives the second order drifts. The $(\nabla \times \vec{u})_{1}$ in (11) may be evaluated explicitly. We have done this and have found no outstanding simplification when all the $O\left(\varepsilon^{2}\right)$ terms were combined. The two terms of $\vec{u}$ are proportional to $M$ and $v_{\|}^{2}$; hence $\left(\frac{d \vec{R}}{d t}\right)$ has in $O\left(\varepsilon^{2}\right)$ terms proportional to $v_{\|} M$ and to $v_{\|}{ }^{3}$, whereas the first order drifts are proportional to even powers of $v_{\|}$. An explicit expression for the drift velocity is

$$
\begin{aligned}
& \left(\frac{d \vec{R}_{0}}{d t}\right)_{\perp}=\varepsilon \frac{M_{0}+\varepsilon M_{1}}{m} \frac{\hat{L} \times \nabla B}{B}+\frac{\varepsilon v_{\|}^{2}}{B} \ddot{L} \times \frac{\partial \tilde{L}}{\partial S} \\
& +\frac{\varepsilon^{2} v_{\|}^{3}}{B^{2}} \hat{L} x\left[\left(\hat{L} \times \frac{\partial \hat{L}}{\partial S}\right) \cdot \nabla \hat{L}+\frac{\partial}{\partial s}\left(\hat{L} \times \frac{\partial \hat{L}}{\partial s}\right)+(\nabla \cdot \hat{L}) \hat{L} \times \frac{\partial \hat{L}}{\partial s}\right] \\
& +\frac{\varepsilon^{2} v_{\|} M}{2 m B^{2}} \hat{L}_{x}\left[2(\hat{L} x \nabla B) \cdot \nabla \hat{L}+2 \frac{\partial}{\partial S}(\hat{L} x \nabla B)+(\nabla \cdot \hat{L}) \hat{L} x\left(3 \nabla B+8 B \frac{\partial \hat{L}}{\partial S}\right)\right. \\
& \left.-2 B \hat{L} \times\left(\frac{\partial \hat{L}}{\partial S} \cdot \nabla \hat{L}\right)-(\hat{L} \cdot \nabla \times \hat{L}) \nabla B-\hat{L} \times\left(\nabla^{2} \vec{B}-\hat{L} \hat{I}: \nabla \nabla \hat{B}\right)\right]+0\left(\varepsilon^{3}\right) \text {, }
\end{aligned}
$$

where $m v_{\|}^{2} / 2=K-\left(M_{0}+\varepsilon M_{1}\right) B$.

For completeness, we can alsn express the magnetic moment in terms of the $\vec{R}_{n}$ variables of (13): 


$$
\frac{M_{0}+\varepsilon M_{1}}{m}=B\left(\vec{R}_{1} \cdot \vec{R}_{1}^{*}\right)+\varepsilon \dot{\vec{R}}_{00} \cdot \nabla \times \hat{L}\left(\vec{R}_{10} \cdot \vec{R}_{10}^{*}\right),
$$

where $\vec{R}_{n}=\vec{R}_{n_{0}}+\varepsilon \vec{R}_{n_{1}}+\cdots$. It must be noted that it is only the sum $M_{0}+\varepsilon M_{1}$ that is conserved through $O(\varepsilon)$ and that the second term which is the $M_{1}$ of Eq. (17) is not the same as the second term of Eq. (2) since the corresponding $M_{0}{ }^{\prime} s$ differ by $O(\varepsilon)$.

We have obtained only the perpendicular drifts and not the parallel velocity through order $\varepsilon^{2}$. That is, we do not have an equation corresponding to Eq. (3), only to one higher order, because we do not have the parallel guiding center velocity through order $\varepsilon^{2}$.

IV. CONSERVATION OF CANONICAL ANGULAR MOMENTUM IN AN AXISYMMETRIC FIELD When the components of $\vec{B}$ are independent of $\phi$ in a $(\rho, \phi, z)$ cylindrical coordinate system, the canonical angular momentum $P_{\phi}=m \rho v_{\phi}+$

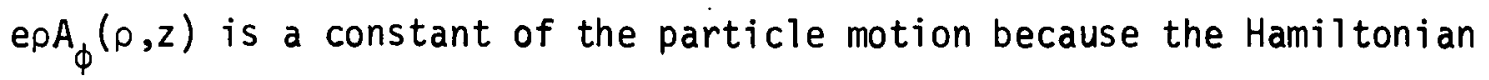
is independent of $\phi$. It is also true that the guiding center velocity $d \vec{R} / d t$ in (3) conserves the $P_{\phi}$ of the guiding center - i.e., that

$$
\frac{d}{d t}\left[\varepsilon \rho v_{\|} \hat{L} \cdot \hat{\phi}+\rho A_{\phi}(\vec{R})\right]=0+0\left(\varepsilon^{2}\right),
$$

where $\rho$ is the guiding center's radial distance from the symmetry axis and $\hat{\phi}$ is the unit vector in the azimuthal direction. Equation (18) is proven directly in Appendix III. The direct proof may be replaced by the following indirect argument: Eq. (8) is identical to the differential equation of motion of a charged particle in a magnetic field $\vec{B}(\vec{R})$ and a potential $M_{0} B(\vec{R})$. We already know that the Hamiltonian for that differential equation is 


$$
\begin{aligned}
H_{\text {guiding center }}=\frac{1}{2 m}\left[\left(P_{\rho}-e A_{\rho}\right)^{2}+\left(P_{z}-e A_{z}\right)^{2}\right. & \left.+\left(\frac{P_{\phi}}{\rho}-e A_{\phi}\right)^{2}\right] \\
& +M_{0} B(\vec{R}),
\end{aligned}
$$

where the momenta are those of the guiding center, and fields are evaluated at the guiding center $\vec{R}$. If $\vec{A}$, hence $B$, is independent of $\phi$ in (19), the usual canonical equations give $\mathrm{dP}_{\phi} / \mathrm{dt}=0+0\left(\varepsilon^{2}\right)$, which is (18). To next order the $\varepsilon \hat{L} \times \vec{W}$ term in (7) destroys the existence of the usual Hamiltonian and hence the invariance of the usual guiding center $P_{\phi}$. In fact,

$$
\frac{d}{d t}\left[\varepsilon \rho \frac{d \vec{R}}{d t} \cdot \hat{\phi}+\rho A_{\phi}(\vec{R})\right]=\varepsilon^{2} \rho(\hat{L} \times \vec{W}) \cdot \hat{\phi}+0\left(\varepsilon^{3}\right),
$$

and so, as expected, the usual $P_{\phi}$ of the guiding center is not conserved to the next order (see Appendix III). We do not now know whether Eq. (7), with either $\vec{W}_{1}$ or any other possible $\vec{W}$, has a locally computable constant of the motion to replace $P_{\phi}$.

Because $\vec{W}$ is proportional to $v_{\|}$and $\frac{d \vec{R}}{d t}=\left[v_{\|}\right.$to lowest order, Eq.

(1) may be written as

$$
\frac{d^{2} \vec{R}}{d t ?}=\frac{1}{\varepsilon} \frac{d \vec{R}}{d t} \times\left[\vec{B}(\vec{R})+\varepsilon^{2} \frac{\vec{W}(\vec{R})}{V_{\|}(\vec{R})}\right]-\frac{M_{0}+\varepsilon M_{1}}{m} \nabla B(\vec{R})+0\left(\varepsilon^{2}\right)
$$

Let us ignore the $(M / m) \nabla B$ term for the moment. (It produces no basic difficulty.) Then (21) is the equation of motion of a charged particle in a continuum fluid of classical magnetic monopoles, distributed so that their (nondivergence-free) magnetic field is $\vec{B}(\vec{R})+\varepsilon^{2} \vec{W}(\vec{R}) / V_{\|}$. Although energy is conserved, because the acceleration is still at right angles to the velocity, and Hamilton's principle still holds, the fact 
that the field has a divergence prevents the formation of the usual Lagrangian and Hamiltonian. Hence, the conclusion that $\mathrm{dP}_{\phi} / \mathrm{dt}=\frac{\partial \mathrm{H}}{\partial \phi}=0$ does not follow for the guiding motion through second order in gyroradius.

\section{CONCLUSION}

We have succeeded in finding the second order drifts, equation of motion, and position for the guiding center. In second order, expressions rapidly become cumbersome and physical insight becomes overwhelmed. To extend the theory further, it seems that the basic definition of the guiding center should be changed so that $P_{\phi}$ would be conserved and (perhaps simultaneous $7 y$ ) $\vec{W}$ would be zero.

For correctness through $0(\varepsilon)$, Eq. (3) for the guiding center velocity should be used with the first order magnetic moment included. If $0\left(\varepsilon^{2}\right)$ results are required, Eq. (14) gives the guiding center, Eq. (16) gives its perpendicular drift velocity, and Eq. (10) gives its acceleration.

\section{ACKNOWLEDGMENTS}

Drs. Martin Kruskal and Steven Hirshman of Princeton University, Thomas J. Birmingham of Goddard, Y-K. M. Peng of Oak Ridge, A. N. Kaufman of the University of California (Berkeley) and R. D. Hazeltine of the University of Texas (Austin) have all contributed to our understanding of matters discussed in this paper. We thank them for their interest and help.. 
The work of James A. Rome was sponsored by the Energy Research and Development Administration under contract with the Union Carbide Corporation.

\section{APPENDIX I \\ STAGNATION ORBITS IN A TOKAMAK}

In a tokamak, guiding center orbits result from a particle trying to follow a field line while being acted upon by the $\nabla B$ and curvature drifts. In the equatorial plane (the $\rho-\phi$ plane of Fig. 1), the poloidal components of these motions are in the z-direction and, for certain orbits, may precisely cancel. These orbits, called "stagnation orbits," move purely in the $\phi$-direction. In some sense these orbits are singular because the area of the guiding center drift surface has shrunk to zero. This singularity is responsible for the geometrical peaking of the fast ion density resulting from neutral beam injection. ${ }^{8}$ Also, these orbits constitute part of the boundary in the constant-of-motion space depiction of tokamak particle orbits. ${ }^{9}$

Because the topology of these orbits is so simple, they are easy to find analytically by two different methods. First, we can use conservation of toroidal canonical angular momentum, $P_{\phi}$ :

$$
\frac{\mathrm{P}_{\phi}}{\mathrm{e}}= \pm \varepsilon \rho \sqrt{\frac{2}{\mathrm{~m}}(K-M B)} \frac{\mathrm{B}_{\phi}}{\mathrm{B}}-\psi+0\left(\varepsilon^{2}\right)
$$

where $\psi$ is the poloidal flux function.

For a fixed $K$ and $M$, the $P_{\phi}=$ constant surfaces each represent an orbit drift surface. The topology of these surfaces in the vicinity of 
the stagnation point is shown in Fig. 1. The stagnation orbit occurs when $\mathrm{dP}_{\phi} / \mathrm{d} \rho=0$ on the equator:

$$
\frac{1}{e} \frac{d P_{\phi}}{d \rho}=\varepsilon v_{\|} \frac{B}{B}+\varepsilon \rho v_{\|} \frac{d}{d \rho}\left(\frac{B}{B}\right)-\frac{M}{m} \cdot \frac{B}{B} \frac{\varepsilon \rho}{v_{\|}} \frac{d B}{d \rho}-\frac{d \psi}{d \rho}+\frac{d}{d \rho}\left[O\left(\varepsilon^{2}\right)\right] .
$$

On the equator, $d \psi / d \rho=\rho B_{z}$, so the condition for a stagnation orbit is

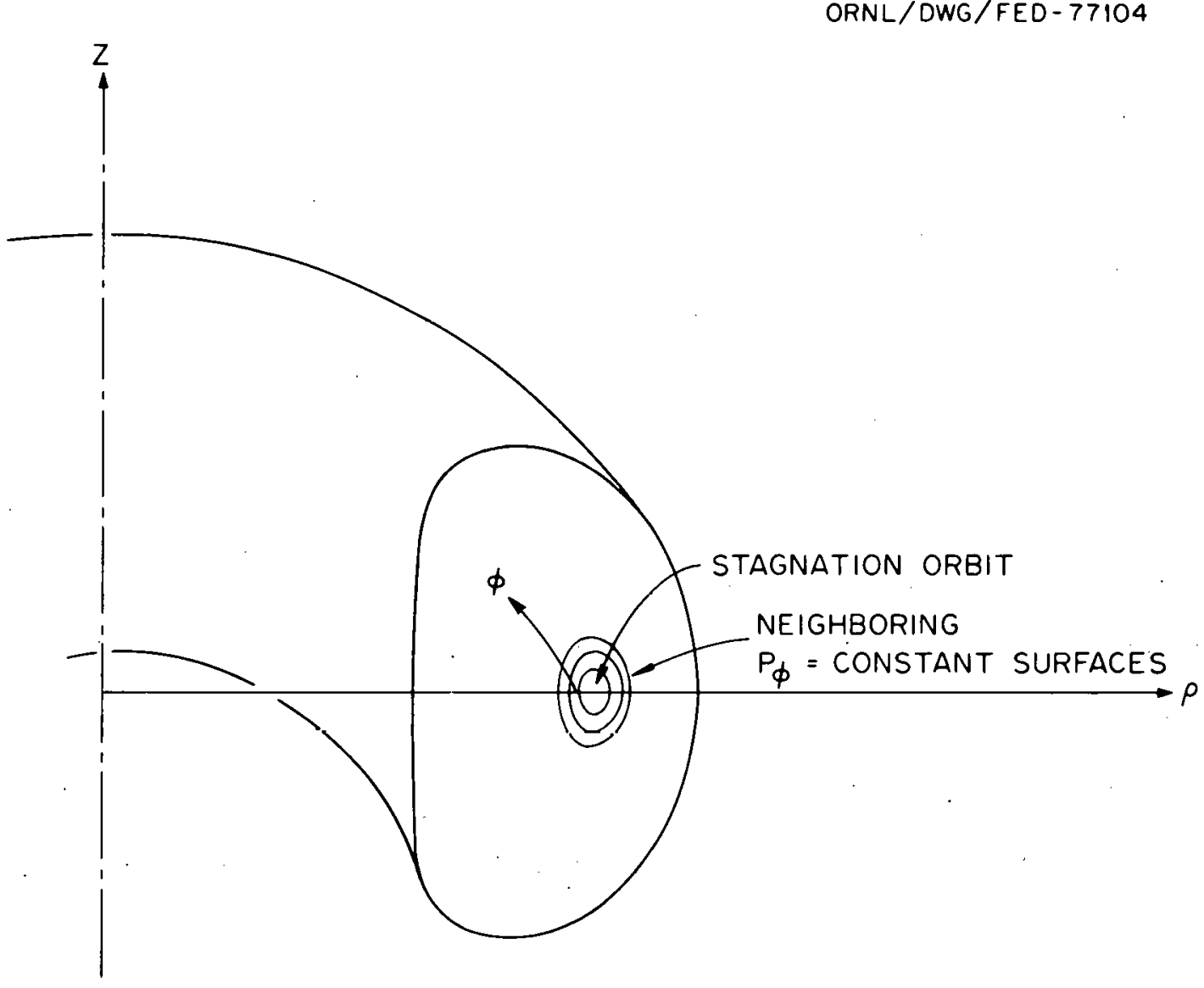

Fig. 1. Geometry of the stagnation orbit. 


$$
v_{\|}^{2} \frac{1}{\rho} \frac{d}{d \rho}\left(\rho \frac{B_{\phi}}{B}\right)-\frac{M}{m} \frac{B_{\phi}}{B} \frac{d B}{d \rho}+\frac{v_{\|} B_{z}}{\varepsilon}=0+0(\varepsilon) .
$$

Also, we should be able to find this orbit by setting the vertical component of the guiding center velocity Eq. (3) to zero. On the equator, $\hat{L} \equiv \hat{z} B z / B+\hat{\phi}_{\phi} / B, \varepsilon 0$

$$
-v_{\|}^{2} \hat{z} \cdot[(\hat{L} \cdot \nabla \times \hat{L}) \hat{L}-\nabla \times \hat{L}]-\frac{M}{m} \frac{B_{\phi}}{B} \frac{d B}{d \rho}+\frac{v_{\|} B}{\varepsilon}=0+0(\varepsilon) .
$$

Since

$$
-v x \hat{L}=\hat{\phi} \frac{d}{d \dot{\rho}}\left(\frac{B_{B}}{B}\right)-\hat{z} \frac{1}{\rho} \frac{d}{d \rho}\left(\frac{\rho B}{B} \phi\right),
$$

we obtain

$$
v_{\|}^{2}\left[\frac{1}{\rho} \frac{d}{d \rho}\left(\frac{\rho B}{B}\right)-\frac{B_{z}}{B} \hat{L} \cdot(\nabla \times \hat{L})\right]-\frac{M}{m} \frac{B}{B} \frac{d B}{d \rho}+\frac{v_{\|} B z}{\varepsilon}=0+0(\varepsilon) .
$$

This differs from the result of the $P_{\phi}$ method by a term proportional to $\mathrm{J}_{\|}:$

$$
v_{\|}^{2} \frac{B}{B} \hat{L} \cdot(\nabla \times \vec{B})
$$

However, since $\mathrm{V}_{\|} \mathrm{B}_{z} / \varepsilon$ must be $0(1)$ in $\mathrm{Eq} .(A 6), \mathrm{B}_{z}$ must be $O(\varepsilon)$, and the difference term is formally of higher order. But, is this difference important?

It is convenient to rewrite Eq. (A6) in terms of other constants of motion. Let $\xi \equiv v_{\|} / v, F \equiv \rho B_{\phi}$, and primes stand for $d / d \psi$.

$$
v=\frac{\omega_{C} \xi}{\xi^{2}\left[\frac{\mu_{0} J_{\|}}{B^{2}}+\left(\frac{F}{B}\right)^{-}\right]-\frac{F B^{-}}{2 B^{2}}\left(1-\xi^{2}\right)}
$$


The $\left(\mu_{0} \mathrm{~J}_{\|} / B^{2}\right)$ term is of order $2 B A$ times its comparison term $(F / B)^{-}$. In most tokamaks the aspect ratio, $A$, is about 4 , so that for high $\beta$ equilibria $(\beta>10 \%)$ this difference is important.

Because the order $\varepsilon^{2}$ term $\left(\varepsilon V_{\|}{ }^{2} B_{z} / B\right) \hat{L} \cdot \nabla \times \vec{B}$ is not as small as one would like, it seems necessary to investigate how much other order $\varepsilon^{2}$ terms, due to second order drifts, would add to (A4). By use of (16) we estimate that here the order $\varepsilon^{2}$ drifts are one to two orders of magnitude less than the terms of (3). Thus, a reasonable procedure for finding the stagnation orbits is to use (A6) as it stands, keeping all three terms. Use of (A3), from conservation of $P_{\phi}$, is not justifiable at nonzero $\beta$ because we do not know what the constant of the guiding center motion is to next order in $\varepsilon$, if indeed there is one. We suspect that $(A 3)$ is not as accurate as (A6) because higher terms of (A6) are indeed small. This can only mean that corrections to (A3) are appreciable, because the difference between the two equations is numerically important. 


\section{APPENDIX II}

\section{BAÑOS' PARALLEL VELOCITY}

Baños ${ }^{6}$ gives the parallel guiding center velocity as

$$
v_{\|} \equiv a_{\|}+\frac{\varepsilon \dot{M_{0}}}{m B}[\cdot \nabla \times \vec{B} \text {, }
$$

where $\bar{u}_{\|}$is the gyration average of the component of particle velocity parallel to the field at the particle. $u_{\|}=\hat{L}(\vec{r}) \cdot \vec{v}$. Equation (B1) says that $\bar{u}_{\|}$differs from $v_{\|}$by order $s$ in a field having parallel current. (B1) does not, however, mean that the particle gradually escapes along the field line from its guiding center. As a simple graphic illustration, consider a $\vec{B}$ field having constant magnitude everywhere with straight but nonparallel field lines as in Fig. 2. Let $\vec{B}$ be independent of $y$,

ORNL / OWG/FED - 77103

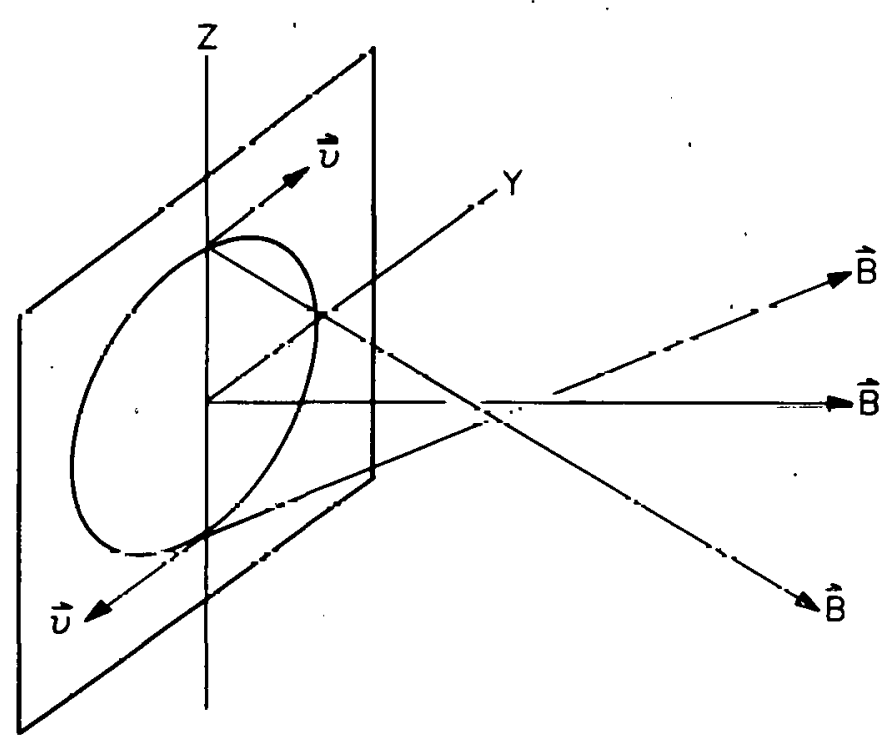

Fig. 2. A sheared field. 
but rotating in direction about $Z$ as one moves along the $Z$ axis: $A$ particle with guiding center at the origin at rest will remain there because $\partial B / \partial s=0$, so there is no acceleration, and the drifts vanish because $\nabla B=0$ and the field lines are straight. Nevertheless, $\hat{L} \cdot \nabla \times \vec{B} \neq 0$, which implies by $(B])$ that $\bar{u}_{\|} \neq 0$. Let the particle gyrate in the circle shown in the figure. $u_{\|}$is in fact negative when the particle is at the top of the circle. A quarter of a rotation later, $u_{\|}$vanishes. The crucial point is that $u_{\|}$is again negative at the bottom of the circle because, even though $\vec{v}$ has reversed from the top of the circle, $\vec{B}$ has deflected the other way. Thus, $u_{\|} \leqslant 0$ throughout the gyration. One can easily show in this simple example that $\bar{u}_{\|}$ $=-\left(\varepsilon M_{0} / m B\right) \hat{L} \cdot \nabla \times \vec{B}$.

\section{APPENDIX III}

\section{DIRECT PROOF THAT THE CANONICAL A ANGULAR MOMENTUM OF THE GUIDING CENTER IS CONSERVED}

A direct proof that $P_{\phi}$ of the guiding center is conserved by the guiding center motion has been given by Morozov and Solov'ev' for the case where $\vec{B} \cdot \nabla \times \vec{B}=0$. Hirshman 10 has shown us a direct proof not subject to this limitation. The following proof is different from his and, like his, does not require that $\vec{B} \cdot \nabla \times \vec{B}=0$. It also establishes (19). By definition of the $P_{\phi}$ of the guiding center,

$$
\frac{P}{e}=\varepsilon \rho \frac{d \vec{R}}{d t} \hat{\phi}+\rho A_{\phi},
$$


where $\rho$ is the distance of the guiding center from the symmetry axis.

$$
\frac{1}{e} \frac{d P_{\phi}}{d t}=\varepsilon \rho \hat{\phi} \cdot \frac{d^{2} \vec{R}}{d t^{2}}+\frac{d \vec{R}}{d t} \cdot \frac{d}{d t}(\hat{\phi} \rho)+\frac{d \vec{R}}{d t} \cdot \nabla\left(\rho A_{\phi}\right) .
$$

In an axisymmetric field, $\nabla\left(\rho A_{\phi}\right)=\rho \hat{\phi} \times \vec{B}$. Substitution of $d^{2} R / d t^{2}$ from (7) gives

$$
\frac{1}{e} \frac{d P}{d t}=\varepsilon^{2} \rho(\hat{L} \times \vec{W}) \cdot \hat{\phi}+\varepsilon\left(\rho \frac{d \vec{R}}{d t} \cdot \frac{d \hat{\phi}}{d t}+\hat{\phi} \cdot \frac{d \vec{R}}{d t} \frac{d \rho}{d t}\right) .
$$

$\hat{\phi} \cdot \nabla B$ has vanished because of the axisymmetry. Now

$$
\frac{d \hat{\phi}}{d t} \equiv \frac{d \vec{R}}{d t} \cdot \nabla \hat{\phi}=\frac{d \vec{R}}{d t} \cdot\left(-\frac{\hat{\phi} \rho}{\rho}\right)=-\frac{\dot{\rho}}{\rho} \hat{\phi} \cdot \frac{d \vec{R}}{d t} \quad,
$$

and $\frac{d \rho}{d t}=\hat{\rho} \cdot d \vec{R} / d t$, so that the last term of (c3) vanishes and

$$
\frac{1}{\mathrm{e}} \frac{\mathrm{dP}}{\mathrm{dt}}=\varepsilon^{2} \rho(\hat{L} \times \vec{W}) \cdot \hat{\phi}+O\left(\varepsilon^{3}\right)
$$

If the right side of (C4) could be shown to be the total time derivative of some quantity, a new invariant through $0\left(\varepsilon^{2}\right)$ would be established. 


\section{REFERENCES}

1. T. G. Northrop, Eq. (1.29) in The Adiabatic Motion of Charged Particles (John Wiley and Sons, New York, 1963).

2. M. Kruskal, "The Gyration of a Charged Particle," Princeton University report PM-S-33 (NYO-7903)(1958) and "Advanced Theory of Gyrating Particles," in Plasma Physics (IAEA, Vienna, 1965), pp. 67-102.

3. T. G. Northrop, C. S. Liu, and M. D. Kruskal, Phys. Fluids 9, 1504 (1966).

4. G. E. Wilson, Phys. Fluids 12, 1673 (1969) and Ph.D. thesis, University of Wisconsin, 1968.

5. A. I. Morozov and L. S. Solov'ev, Reviews of Plasma Physics 2, 201 (1966), ed. by M. A. Leontovich.

6. A. Baños, J. Plasma Phys. 1, 305 (1967).

7. M. Kruska7, J. Math. Phys. 3, 806 (1962).

8. J. A. Rome, J. D. Callen, and J. F. Clarke, Nucl. Fusion 14, 141 (1974).

9. J. A. Rome and Y-K. M. Peng, Bul1. Am. Phys. Soc. 9, 1100 (1976).

10. Steven P. Hirshman (PPPL), private communication. 


\section{THIS PAGE}

\section{WAS INTENTIONALLY}

LEFT BLANK 
ORNL/TM-5952

INTERNAL DISTRIBUTION

1. J. D. Callen

2. J. F. Clarke

3. R. A. Dory

4. G. G. Kelley

5. 0. B. Morgan

6. M. W. Rosenthal

7-31. J. A. Rome

32-34. Laboratory Records Department

35. Laboratory Records, ORNL - RC

36. Y-12 Document Reference Section

37-38. Central Research Library

39. Fusion Energy Division Library

40. Fusion Energy Division Reports Office

41. ORNL Patent Office

\section{EXTERNAL DISTRIBUTION}

42. Plasma Physics Library, Plasma Physics Laboratory, Princeton Univ., Forrestal Campus, P.0. Box 451, Princeton, NJ 08540

43. Controlled Thermonuclear Research Library, Lawrence Livermore Laboratory, P.0. Box 808, Livermore, CA 94550

44. Q Division Library, Los Alamos Scientific Laboratory, P.0. Box 1663, Los A7amos, NM 87544

45. Controlled Thermonuclear Research Library, c/o Weston M. Stacey, Jr., Argonne National Laboratory, 9700 S. Cass Ave., Argonne, IL 60439

46. CTR Computer Center, C/O Dr. John Killeen, Lawrence Livermore Laboratory, P.0. Box 808, Livermore, CA 94550

47. Librarian, Culham Laboratory, U.K. Atomic Energy Authority, Abingdon, Oxon, 0X14 3DB, United Kingdom

48. Ruth Lengye, Bibliothek, Max-Planck Institut für Plasmaphysik, 8046 Garching bei München, Federal Republ ic of Germany

49. Library, Centre de Recherches en Physique des Plasmas, 21 Avenue des Bains, 1007, Lausanne, Switzerland

50. A. M. Dupas, Documentation S.I.G.N., Department de 1a Physique du Plasma et de la Fusion Controlé, Association EURATOM-CEA sur 1a Fusion, Centre d'Etudes Nucleaires, BP 85 Centre Du TRI 38041 Grenoble Cedex (France)

51. Bibliothèque, Service du Confinement des Plasmas, C.E.A., B.P. No. 6,92 , Fontenay-aux-Roses (Seine) France

52. Library, International Centre for Theoretical Physics, Trieste, Italy

53. Library, Laboratorio Gas Ionizzati, Frascati, Italy

54. V. E. Ivanov, Physical-Technical Institute of the Ukranian Academy of Sciences, Sukhumi, U.S.S.R.

55. L. M. Kovrizhnikh, Lebedev Institute of Physics, Academy of Sciences of the U.S.S.R., Leninsky Prospect 53, Moscow, U.S.S.R.

56. Prof. Dshumber G. Lominadze, Academy of Sciences of the Georgian SSR, 8 Dzerżhinski St., 38004, Tbilisi, U.S.S.R.

57. Library, Inst. for Plasma Physics, Nagoya Univ., Nagoya, Japan 464 
58. Library, FOM-Institut voor Plasma-Fysica, Rijnhuizen, Jutphaas, Netherlands

59. Plasma Physics Group, Department of Engineering Physics, Australian National University, P.0. Box 4, Canberra A.C.T. 2600, Australia

60. Thermonuclear Library, Japan Atomic Energy Research Institute, Tokai, Naka, Ibaraki, Japan

61. Dr. D. G. McAlees, Exxon Nuclear Co., Inc., Research \& Technology Laser Enrichment Department, 2955 George Washington Way, Richland, WA 99352

62. CTR Reading Room, c/o Prof. D. W. Kerst, Dept. of Physics, Sterling Hal1, Univ. of Wisconsin, Madison, WI 53706

63. CTR Reading Room, c/o Prof. I. B. Bernstein, Yale Univ., 200 Mason Laboratory, Dept. of Engineering \& Applied Science, New Haven, CT 06510

64. Center for Plasma Physics and Thermonuclear Research, c/o D. W. Ross, Physics Dept. . Univ. of Texas, Austin, TX 78712

65. CTR Reading Room, c/o Prof. B. D. Fried, Physics Dept., Univ. of California, Los Angeles, CA 90024

66. CTR Reading Room, c/o Prof. David C. Montgomery, Physics \& Astronomy Dept., Univ. of Iowa, Iowa City, IA 52240

67. Magneto-Fluid-Dynamics Library, c/o Dr. Harold Grad, Courant Inst. of Math. Sci., New York Univ., 251 Mercer St., New York, NY 10012

68. CTR Reading Room, c/o Prof. Allan N. Kaufman, Physics Dept., Univ. of California, Berkeley, CA 94720

69. Dr. David A. Dingee, Fusion Programs, Battelle-Northwest, Battelle Boulevard, Richland, WA 99352

70. CTR Reading Room, c/o Prof. C. S. Liu, Dept. of Physics and Astronomy, Univ, of Maryland, College Park, MD 20742

71. CTR Reading Room, c/0 Prof. T. Kammash, 103 Research Admin. Bldg., N. Campus, Univ. of Michigan, Ann Arbor, MI 48105

72. CTR Reading Room, c/o Dr. Ravi N. Sudan, Phillips Hall, Cornell Univ., Ithaca, NY 14850

73. Prof. Marshali N. Rosentluth, Institute Für Advallced Sludy, Princeton; NJ 08540

74. CTR Reading Room, c/o Prof. R. Gross, Plasma Research Lab., Columbia Univ., New York, NY 10027

75. CTR Reading Room, c/o Prof. Roy Gould, California Inst. of Tech., M.S. 116-81, Pasadena, CA 91125

76. Dr. Nicholas A. Krall, Science Applications, Inc., P.0. Box 2354, 1200 Prospect St., La Jolla, CA 92037

77. CTR Reading Room, c/o Dr. Jay P. Boris, Plasma Physics, Naval Research Laboratory, Washington, D.C. 20390

78. Professor A. Simon, Dept. of Mechanical \& Aerospace Sciences, Universily of Ruche'sler, Rucherster, NY 14627

79. CTR Library, c/o Dr. Alan F. Haught, United Technologies Research Labs, East Hartford, CT 06108

80. Dr. H. K. Forsen, Exxon Nuclear Co., Inc., 777 106th Avenue, NE, C-000777, Bellevue, WA 98009

81. Dr. George Vahala, Physics Dept., College of William \& Mary, Williamsburg, VA 23185

82. Dr. Robert E. Price, Division of Magnetic Fusion Energy, G-234, Energy Research and Development Administration, Washington, D.C. 20545 
83. Dr. R. C. Davidson, Division of Magnetic Fusion Energy, G-234, Energy Research and Development Administration, Washington, D.C. 20545

84. Dr. Oscar P. Manley, Division of Magnetic Fusion Energy, G-234, Energy Research and Development Administration, Washington, D.C. 20545

85. Mr. E. E. Kintner, Division of Magnetic Fusion Energy, G-234, Energy Research and Development Administration, Washington, D.C. 20545

86. Dr. L. D. Pearlstein, L-388, Lawrence Livermore Laboratory, P.0. Box 808, Livermore, CA 94550

87. Dr. J. P. Friedberg, Los Alamos Scientific Laboratory, Los Alamos, NM 87544

88. Dr. David J. Rose, Dept. of Nuclear Engineering, MIT, Cambridge, MA 02139

89. Dr. Gareth E. Guest, General Atomic Co., P.0. Box 81608, San Diego, CA 92138

90. Dr. Claude Mercier, Service du Theorie des Plasmas, Centre d'Etudes Nucléaires, Fontenay-aux-Roses (Seine) France

91. Dr. J. B. Taylor, Culham Laboratory, UKAEA, Abingdon, Oxon, $0 \times 14$ 3DB, United Kingdom

92. Dr. D. Pfirsch, Institute for Plasma Physics, 8046 Garching bei München, Federal Republic of Germany

93. Dr. V. D. Shafranov, I. V. Kurchatov Inst. of Atomic Energy, 46 Ulitsa Kurchatova, P.0. Box 3402, Moscow, U.S.S.R.

94. Dr. A. Rogister, Institute for Plasma Physics, KFA, Postfach 1913, D-5170, Jülich 1, Federal Republic of Germany

95. Dr. J. G. Cordey, Culham Laboratory, UKAEA, Abingdon, 0xon, 0X14 3DB, United Kingdom

96. Dr. David Baldwin, L-388, Lawrence Livermore Lab., P.0. Box 808, Livermore, CA 94550

97. CTR Reading Room, c/o Prof. Bruno Coppi, Dept. of Physics, MIT, Cambridge, MA 02138

98. Dr. Harold P. Furth, Princeton Plasma Physics Lab., Princeton Univ., P.0. Box 451, Princeton, NJ 08540

99. Dr. Paul H. Rutherford, Princeton Plasma Physics Lab., Princeton Univ., P.0. Box 451, Princeton, NJ 08540

100. Research and Technical Support Division, Energy Research and Development Administration, P.0. Box E, Oak Ridge, TN 37830

101-150. Theodore G. Northrup, NASA/Goddard Space Flight Center, Greenbelt, MD 20771

151-177. Technical Information Center, P.0. Box 62, Oak Ridge, TN 37830 